\title{
Viscous potential flow analysis of magnetohydrodynamic Rayleigh-Taylor instability with heat and mass transfer
}

\author{
Mukesh Kumar Awasthi
}

Received: 27 May 2013 / Revised: 1 December 2013 / Accepted: 5 December 2013 / Published online: 19 December 2013

(C) Springer-Verlag Berlin Heidelberg 2013

\begin{abstract}
The effect of tangential magnetic field on the linear analysis of Rayleigh-Taylor instability of two viscous, incompressible and electrically conducting fluids is studied when there is heat and mass transfer across the interface. We use an irrotational theory known as viscous potential flow theory; in which viscosity enters through normal stress balance but shearing stresses are assumed to be zero. A quadratic dispersion relation that accounts for the growth of disturbance waves is obtained and stability criterion is given in terms of a critical value of wave number as well as applied magnetic field. We observe that heat and mass transfer and magnetic field both stabilize the interface while vapour thickness has destabilizing effect.
\end{abstract}

Keywords Viscous potential flow - Rayleigh-Taylor instability · Magnetic field · Incompressible fluid .

Viscous stresses

\section{List of symbols}

$\begin{array}{ll}\rho^{(1)} & \text { Density of the lower fluid } \\ \rho^{(2)} & \text { Density of the upper fluid } \\ \mu^{(1)} & \text { Viscosity of the lower fluid } \\ \mu^{(2)} & \text { Viscosity of the upper fluid } \\ \mu_{m}^{(1)} & \text { Magnetic permeability of lower fluid } \\ \mu_{m}^{(2)} & \text { Magnetic permeability of upper fluid } \\ h_{1} & \text { Thickness of the lower fluid } \\ h_{2} & \text { Thickness of the upper fluid } \\ \sigma & \text { Surface tension at the interface }\end{array}$

M. K. Awasthi $(\varangle)$

Department of Mathematics, University of Petroleum and Energy Studies, Dehradun 248007, India

e-mail: mukeshiitr.kumar@gmail.com

$\begin{array}{ll}\eta & \text { Perturbation from equilibrium state } \\ \boldsymbol{n} & \text { Unit outward normal to the interface } \\ k & \text { Wave number } \\ \phi(x, y, t) & \text { Velocity potential function } \\ \omega & \text { Growth rate } \\ \psi_{m}(x, y, t) & \text { Magnetic potential function } \\ H_{0} & \text { Magnetic field intensity }\end{array}$

\section{Introduction}

The instability of the interface separating two fluids having different densities when the lighter fluid is accelerated toward the heavier fluid is known as Rayleigh-Taylor instability [1, 2]. Rayleigh-Taylor instability is buoyancy-driven instability and occurs in several types of fluid flows that contain different variety of molecular masses in the presence of acceleration, such as gravity or accelerating fronts [3].

The heat and mass transfer phenomenon in multiphase flows has received much attention in recent years because of its wide range of applications in many situations such as boiling heat transfer in chemical engineering and in geophysical problems. The general formulation of interfacial flow problem of two inviscid fluids with heat and mass transfer for Rayleigh-Taylor and Kelvin-Helmholtz instabilities in plane geometry was established by Hsieh [4,5]. Hsieh [5] found that when the vapour layer is hotter than the liquid layer, the effect of heat and mass transfer tends to inhibit the growth of instability.

The linear analysis of Rayleigh-Taylor instability of two viscous fluids of same kinematic viscosities in the presence of heat and mass transfer was considered by Ho [6]. He observed that heat and mass transfer phenomenon plays stabilizing role in the stability criterion. Khodaparast et al. [7] studied the Rayleigh-Taylor and Kelvin-Helmholtz stability 
of liquid-vapour interface and considered liquid as viscous and motionless and vapour as inviscid moving with a horizontal velocity. They observed that coupled viscosity-phase change has stabilizing effect on Rayleigh-Taylor stability whereas it has destabilizing effect on Kelvin-Helmholtz instability.

In viscous potential flow, the flow is irrotational flow and therefore, the viscous term i.e. $\mu \nabla^{2} \boldsymbol{u}$ in the Navier-Stokes equation is identically zero because vorticity is zero but the viscous stresses are not zero, where $\mu$ denotes the viscosity and $\boldsymbol{u}$ denotes the velocity of the fluid flow. There exists a pressure difference across the interface. We include normal stress for calculating this pressure difference and the viscosity enters through the normal stress balance (Joseph and Liao [8]). Joseph et al. [9] considered the viscous potential flow analysis of Rayleigh-Taylor instability and observed that the most dangerous wave is the one whose length gives the maximum growth rate. Funada and Joseph [10] used the viscous potential flow theory to study the Kelvin-Helmholtz instability in a channel and found that the stability criterion for viscous potential flow is given by the critical value of the relative velocity.

Asthana and Agrawal [11] studied the effect of heat and mass transfer phenomenon on the viscous potential flow analysis of Kelvin-Helmholtz instability. They observed that heat and mass transfer has a strong stabilizing effect when the lower fluid is highly viscous and a weak destabilizing effect when the fluid's viscosity is low. The viscous potential flow analysis of Rayleigh-Taylor instability in the presence of heat and mass transfer was carried out by Awasthi and Agrawal [12]. They observed that heat and mass transfer has stabilizing effect.

Magnetohydrodynamics is the study of the interaction between magnetic fields and fluid conductors of electricity. Heat and mass transfer phenomenon has played an important role in the stability of the magnetic fluids. MHD interfacial stability with heat and mass transfer is of fundamental importance in number of applications such as design of many types of contacting equipment, e.g., boilers, condensers, reactors, and others in industrial and environmental processes. Bubble formation in superposed magnetic fluids in the presence of heat and mass transfer has been studied by Gill et al. [13]. Moatimid and El-Dib [14] investigated the stability of an interface between two inviscid magnetic fluids of different densities flowing parallel to each other in an oscillatory manner under the influence of an oblique magnetic field and heat and mass transfer. Elhefnawy [15] considered the nonlinear stability of ferromagnetic fluids with mass and heat transfer and showed that mass and heat transfer coefficient play an important role in the nonlinear stability of the system. Stability of two rigidly rotating magnetic fluid columns in zero gravity in the presence of mass and heat transfer has been carried out by Moatimid [16].
Sirwah [17] applied viscous potential flow theory to study the Kelvin-Helmholtz instability in the presence of tangential magnetic field and observed that the tangential magnetic field plays a stabilizing role in the stability analysis. Sirwah [18] studied the nonlinear stability analysis of magnetic fluids in the presence of constant normal magnetic field and observed that normal magnetic field has destabilizing effect on the stability of the system. Awasthi and Agrawal [19] studied the viscous contribution to the pressure for the potential flow analysis of Kelvin-Helmholtz instability in the presence of tangential magnetic field and observed that the inclusion of irrotational shearing stresses stabilize the system.

Recently, Awasthi et al. [20] carried out the viscous corrections for the potential flow analysis of Kelvin-Helmholtz instability of two viscous fluids in the presence of tangential magnetic field when there is heat and mass transfer across the interface and observed that irrotational shearing stresses have stabilizing effect.

To, the best of our knowledge, the combined effect of magnetic field and heat and mass transfer on the RayleighTaylor instability of two viscous fluids in the plane geometry is not investigated, yet. Hence, an attempt has been made to study the effect of tangential magnetic field on the viscous potential flow analysis of Rayleigh-Taylor instability when there is heat and mass transfer across the interface. Both fluids are taken as incompressible, viscous and electrically conducting with different kinematic viscosities and magnetic permeability's. A quadratic dispersion relation is derived and a critical value of wave number as well as magnetic field is obtained. The effects of various physical parameters such as magnetic field strength, ratio of magnetic permeability of two fluids and viscosity ratio are studied. It is observed that heat and mass transfer and tangential magnetic field both play stabilizing role on the stability of the system. Finally, a comparison has been made between the results of present study with the results obtained by Awasthi and Agrawal [12] for viscous potential analysis.

\section{Problem formulation}

Consider the parallel flow of two incompressible, electrically conducting and viscous fluid layers of finite thickness separated by an interface $y=0$, as demonstrated in Fig. 1. In the formulation, the superscripts 1 and 2 denote the variables associated with the lower fluid and upper fluid, respectively. Initially, lower fluid of density $\rho^{(1)}$, viscosity $\mu^{(1)}$ and magnetic permeability $\mu_{m}^{(1)}$ occupies the region $-h_{1}<y<0$ and upper fluid of density $\rho^{(2)}$, viscosity $\mu^{(2)}$ and magnetic permeability $\mu_{m}^{(2)}$ occupies the region $0<y<h_{2}$. The bounding surfaces $y=-h_{1}$ and $y=h_{2}$ are considered to be rigid. The temperatures at surfaces $y=-h_{1}, y=0$ and $y=h_{2}$ are taken as $T_{1}, T_{0}$ and $T_{2}$, respectively. We have assumed that 


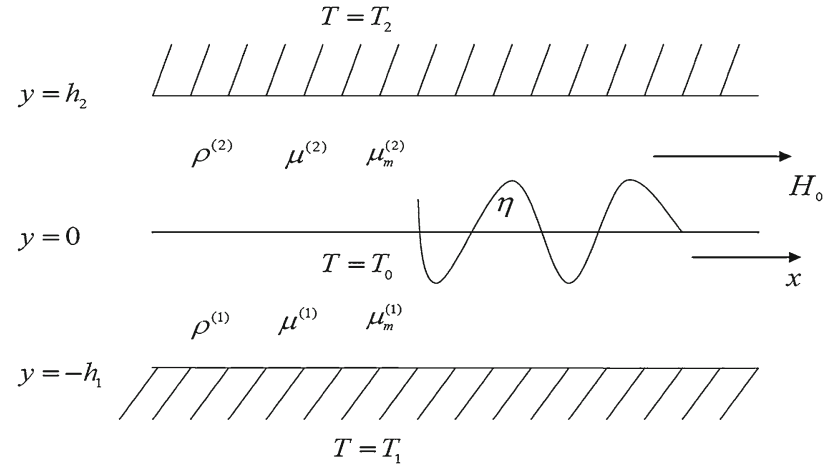

Fig. 1 Equilibrium configuration of the system

the fluid flows are irrotational. In the basic state, thermodynamics equilibrium is assumed and the interface temperature $T_{0}$ is set equal to the saturation temperature.

On applying the small disturbances to the equilibrium state, the equation of interface can be written as

$F(x, y, t)=y-\eta(x, t)=0$

where $\eta$ is the perturbation from its equilibrium value, and for which the outward unit normal vector is given by

$\boldsymbol{n}=\left(-\frac{\partial \eta}{\partial x} \boldsymbol{e}_{\boldsymbol{x}}+\boldsymbol{e}_{\boldsymbol{y}}\right)$

where $\boldsymbol{e}_{\boldsymbol{x}}$ and $\boldsymbol{e}_{\boldsymbol{y}}$ are unit vectors along the $x$ and $y$ directions, respectively.

Our analysis is based on the potential flow theory, therefore; velocity can be expressed as the gradient of the potential function i.e. $\boldsymbol{u}=\nabla \phi$ and for incompressible fluids, the density is constant; the continuity equation takes the form as

$\nabla \cdot \boldsymbol{u}_{j}=0$

Combining the equations, we have

$\nabla^{2} \phi^{(j)}=0, \quad j=1,2$

where $\nabla^{2}=\frac{\partial^{2}}{\partial x^{2}}+\frac{\partial^{2}}{\partial y^{2}}$.

The two fluids are subjected to a uniform magnetic field $H_{0}$, acting along $x$-axis i.e.

$\boldsymbol{H}=H_{0} \mathbf{e}_{x}$.

It is assumed that the quasi-static approximation is valid for this problem and therefore, the magnetic field can be derived from magnetic scalar potential function $\psi_{m}(x, y, t)$ such that

$\boldsymbol{H}_{j}=H_{0} \boldsymbol{e}_{\boldsymbol{x}}-\nabla \psi_{m}^{(j)}, \quad j=1,2$.

Gauss's law requires that the magnetic potentials also satisfy the Laplace's equation i.e.

$\nabla^{2} \psi_{m}^{(j)}=0, \quad j=1,2$.
The boundary conditions at the rigid surfaces $y=-h_{1}$ and $y=h_{2}$ can be written as

$$
\begin{aligned}
& \left(\frac{\partial \phi^{(1)}}{\partial y}\right)=0 \quad \text { at } \quad y=-h_{1}, \\
& \left(\frac{\partial \phi^{(2)}}{\partial y}\right)=0 \quad \text { at } \quad y=h_{2} .
\end{aligned}
$$

and

$\left(\frac{\partial \psi_{m}^{(1)}}{\partial x}\right)=0 \quad$ at $\quad y=-h_{1}$,

$$
\left(\frac{\partial \psi_{m}^{(2)}}{\partial x}\right)=0 \quad \text { at } \quad y=h_{2},
$$

The interfacial condition, which expresses the conservation of mass across the interface, is given by the equation

$$
\begin{aligned}
& {\left[\left[\rho\left(\frac{\partial F}{\partial t}+\nabla \phi \cdot \nabla F\right)\right]=0\right.} \\
& \quad \text { or }\left[\left[\rho\left(\frac{\partial \phi}{\partial y}-\frac{\partial \eta}{\partial t}-\frac{\partial \eta}{\partial x} \frac{\partial \phi}{\partial x}\right)\right]\right]=0 \text { at } y=\eta
\end{aligned}
$$

where $\llbracket x \rrbracket=x^{(2)}-x^{(1)}$ in which superscripts 2 and 1 refer to upper and lower fluids, respectively. Since there is no interfacial material source, they naturally should be equal.

The tangential component of the magnetic field must be continuous across the interface, i.e. $\boldsymbol{n} \times \llbracket \boldsymbol{H} \rrbracket=\mathbf{0}$, or

$\left.\left.\left.\llbracket \frac{\partial \psi_{m}}{\partial x}\right]\right]+\frac{\partial \eta}{\partial x} \llbracket\left[\frac{\partial \psi_{m}}{\partial y}\right]\right]=0$

There is a discontinuity in the normal current across the interface; charge accumulation within a material element is balanced by conduction from bulk fluid on either side of the surface. The boundary condition, corresponding to normal component of magnetic field, at the interface is given by

$$
\begin{aligned}
& \boldsymbol{n} \cdot \llbracket \mu_{m} \boldsymbol{H} \rrbracket=0 \quad \text { or } \\
& \left.\left.\left.H_{0} \frac{\partial \eta}{\partial x} \llbracket \mu_{m} \rrbracket-\frac{\partial \eta}{\partial x} \llbracket \llbracket \mu_{m} \frac{\partial \psi_{m}}{\partial x}\right]\right]+\llbracket\left[\mu_{m} \frac{\partial \psi_{m}}{\partial y}\right]\right]=0
\end{aligned}
$$

In the present analysis, we have assumed that the amount of latent heat released depends mainly on the instantaneous position of the interface. Therefore, the interfacial condition for energy transfer is expressed as

$$
L \rho^{(1)}\left(\frac{\partial F}{\partial t}+\nabla \phi^{(1)} \cdot \nabla F\right)=S(\eta) \quad \text { at } \quad y=\eta
$$

where $L$ is the latent heat released during phase transformation and $S(\eta)$ denotes the net heat flux from the interface.

In the equilibrium state, the heat fluxes in positive $y$-direction in the fluid phase 1 and phase 2 are $K_{1}\left(T_{1}-T_{0}\right) /$ $h_{1}$ and $K_{2}\left(T_{0}-T_{2}\right) / h_{2}$, respectively where $K_{1}$ and $K_{2}$ 
denote heat conductivities of the two fluids. The expression for $S(y)$ can be written as follows

$S(y)=\frac{K_{2}\left(T_{0}-T_{2}\right)}{h_{2}-y}-\frac{K_{1}\left(T_{1}-T_{0}\right)}{h_{1}+y}$

On expanding $S(\eta)$ in the neighbourhood of $\eta=0$, we get

$S(\eta)=S(0)+\eta S^{\prime}(0)+\frac{1}{2} \eta^{2} S^{\prime \prime}(0)+\frac{1}{6} \eta^{3} S^{\prime \prime \prime}(0)+\cdots$

Since $S(0)=0$ from the equilibrium condition, we obtain from Eq. (16)

$$
\frac{K_{2}\left(T_{0}-T_{2}\right)}{h_{2}}=\frac{K_{1}\left(T_{1}-T_{0}\right)}{h_{1}}=G \text { (say) }
$$

The interfacial normal stress is balanced at the dividing surface of the system, so we have

$$
\begin{aligned}
\rho^{(1)} & \left(\nabla \phi^{(1)} \cdot \nabla F\right)\left(\frac{\partial F}{\partial t}+\nabla \phi^{(1)} \cdot \nabla F\right) \\
= & \rho^{(2)}\left(\nabla \phi^{(2)} \cdot \nabla F\right)\left(\frac{\partial F}{\partial t}+\nabla \phi^{(2)} \cdot \nabla F\right) \\
& +\left(p_{2}-p_{1}-2 \mu^{(2)} \boldsymbol{n} \cdot \nabla \otimes \nabla \phi^{(2)} \cdot \boldsymbol{n}\right. \\
& +2 \mu^{(1)} \boldsymbol{n} \cdot \nabla \otimes \nabla \phi^{(1)} \cdot \boldsymbol{n} \\
& \left.\left.-\frac{1}{2}\left[\mu_{m}\left(H_{n}^{2}-H_{t}^{2}\right)\right]\right]+\sigma \nabla \cdot \boldsymbol{n}\right)|\nabla F|^{2}
\end{aligned}
$$

where $p_{j}(j=1,2)$ are the irrotational pressure for lower and upper fluid, respectively and $\sigma$ denotes the surface tension.

\section{Linearization and normal mode analysis}

To, study the stability of the system, small disturbances are superimposed on the Eqs. (12)-(15) and Eq. (19) and retaining the linear terms we can get the following equations

$$
\begin{aligned}
& \llbracket\left[\rho\left(\frac{\partial \phi}{\partial y}-\frac{\partial \eta}{\partial t}\right)\right]=0 \\
& \left.\llbracket\left[\frac{\partial \psi_{m}}{\partial x}\right]\right]=0 \\
& \llbracket\left[\mu_{m}\left(\frac{\partial \psi_{m}}{\partial y}+H_{0} \frac{\partial \eta}{\partial x}\right)\right]=0 \\
& \rho^{(1)}\left(\frac{\partial \phi^{(1)}}{\partial y}-\frac{\partial \eta}{\partial t}\right)=\alpha \eta \\
& {\left[\left[\rho\left(\frac{\partial \phi}{\partial t}+g \eta\right)+2 \mu \frac{\partial^{2} \phi}{\partial y^{2}}+\mu_{m} H_{0} \frac{\partial \psi_{m}}{\partial x}\right]\right]=-\sigma \frac{\partial^{2} \eta}{\partial x^{2}}}
\end{aligned}
$$

where the parameter $\alpha=\frac{G}{L}\left(\frac{1}{h_{2}}+\frac{1}{h_{1}}\right)$ is obtained using Eqs. (15)-(18).
Now the normal mode technique has been used to find the solution of the governing equations. Let the interface elevation be represented by

$\eta=A \exp (i k x-i \omega t)+c . c$.

where $A$ represents the amplitude of the surface wave, $k$ is the real wave number, $\omega$ is the growth rate and c.c. refers the complex conjugate of the preceding term.

Now using normal mode analysis and using the boundary conditions, the solution of Eqs. (4) and (7) can be written as

$$
\begin{aligned}
\phi^{(1)}= & \frac{1}{k}\left(\frac{\alpha}{\rho^{(1)}}-i \omega\right) A \frac{\cosh \left(k\left(y+h_{1}\right)\right)}{\sinh \left(k h_{1}\right)} \exp (i k x-i \omega t) \\
& +c . c . \\
\phi^{(2)}= & -\frac{1}{k}\left(\frac{\alpha}{\rho^{(2)}}-i \omega\right) A \frac{\cosh \left(k\left(y-h_{2}\right)\right)}{\sinh \left(k h_{2}\right)} \exp (i k x-i \omega t) \\
& + \text { c.c. } \\
\psi_{m}^{(1)}= & \frac{i H_{0}\left(\mu_{m}^{(2)}-\mu_{m}^{(1)}\right)}{\left(\mu_{m}^{(1)} \operatorname{coth}\left(k h_{1}\right)+\mu_{m}^{(2)} \operatorname{coth}\left(k h_{2}\right)\right)} \\
& \times A \frac{\sinh \left(k\left(y+h_{1}\right)\right)}{\sinh \left(k h_{1}\right)} \exp (i k x-i \omega t)+c . c \\
\psi_{m}^{(1)}= & -\frac{i H_{0}\left(\mu_{m}^{(2)}-\mu_{m}^{(1)}\right)}{\left(\mu_{m}^{(1)} \operatorname{coth}\left(k h_{1}\right)+\mu_{m}^{(2)} \operatorname{coth}\left(k h_{2}\right)\right)} \\
& \times A \frac{\sinh \left(k\left(y-h_{2}\right)\right)}{\sinh \left(k h_{2}\right)} \exp (i k x-i \omega t)+c . c
\end{aligned}
$$

\section{Dispersion relation}

We have used the expressions of $\eta, \phi^{(1)}, \phi^{(2)}, \psi^{(1)}$ and $\psi^{(2)}$ in Eq. (24) to find the dispersion relation which is a quadratic equation expressed as follows

$D(\omega, k)=a_{0} \omega^{2}+i a_{1} \omega+a_{2}=0$

where

$$
\begin{aligned}
a_{0}= & \rho^{(1)} \operatorname{coth}\left(k h_{1}\right)+\rho^{(2)} \operatorname{coth}\left(k h_{2}\right) \\
a_{1}= & \alpha\left(\operatorname{coth}\left(k h_{1}\right)+\operatorname{coth}\left(k h_{2}\right)\right)+4 k^{2}\left(\mu^{(1)} \operatorname{coth}\left(k h_{1}\right)\right. \\
& \left.+\mu^{(2)} \operatorname{coth}\left(k h_{2}\right)\right) \\
a_{2}= & \left(\rho^{(2)}-\rho^{(1)}\right) g k-\sigma k^{3} \\
& -2 k^{2} \alpha\left(\frac{\mu^{(1)}}{\rho^{(1)}} \operatorname{coth}\left(k h_{1}\right)+\frac{\mu^{(2)}}{\rho^{(2)}} \operatorname{coth}\left(k h_{2}\right)\right) \\
& -\frac{k^{2} H_{0}^{2}\left(\mu_{m}^{(2)}-\mu_{m}^{(1)}\right)^{2}}{\left(\mu_{m}^{(1)} \operatorname{coth}\left(k h_{1}\right)+\mu_{m}^{(2)} \operatorname{coth}\left(k h_{2}\right)\right)}
\end{aligned}
$$

Let $\omega=\omega_{R}+i \omega_{I}$, and separating the real and imaginary parts of Eq. (30), we have

$a_{0}\left(\omega_{R}^{2}-\omega_{I}^{2}\right)-a_{1} \omega_{I}+a_{2}=0$ 
and

$2 a_{0} \omega_{R} \omega_{I}+a_{1} \omega_{R}=0$

So

$\omega_{R}=0$

Putting the above value of $\omega_{R}$ in Eq. (31), we obtained a quadratic equation in $\omega_{I}$ as

$$
\begin{aligned}
& {\left[\rho^{(1)} \operatorname{coth}\left(k h_{1}\right)+\rho^{(2)} \operatorname{coth}\left(k h_{2}\right)\right] \omega_{I}^{2}} \\
& +\left[\alpha\left(\operatorname{coth}\left(k h_{1}\right)+\operatorname{coth}\left(k h_{2}\right)\right)\right. \\
& \left.+4 k^{2}\left(\mu^{(1)} \operatorname{coth}\left(k h_{1}\right)+\mu^{(2)} \operatorname{coth}\left(k h_{2}\right)\right)\right] \omega_{I} \\
& +\left[\left(\rho^{(1)}-\rho^{(2)}\right) g k+\sigma k^{3}\right. \\
& +2 k^{2} \alpha\left(\frac{\mu^{(1)}}{\rho^{(1)}} \operatorname{coth}\left(k h_{1}\right)+\frac{\mu^{(2)}}{\rho^{(2)}} \operatorname{coth}\left(k h_{2}\right)\right) \\
& \left.+\frac{k^{2} H_{0}^{2}\left(\mu_{m}^{(2)}-\mu_{m}^{(1)}\right)^{2}}{\left(\mu_{m}^{(1)} \operatorname{coth}\left(k h_{1}\right)+\mu_{m}^{(2)} \operatorname{coth}\left(k h_{2}\right)\right)}\right]=0
\end{aligned}
$$

If the fluids are considered to be inviscid i.e. $\mu^{(1)}=\mu^{(2)}=$ 0 , Eq. (33) reduces to the same dispersion relation as obtained by Elhefanwy [15]. If there is no magnetic field i.e. $H_{0}=0$, the dispersion relation reduces to the same dispersion relation as obtained by Awasthi and Agrawal [12].

On application of the Routh-Hurwitz criteria in the Eq. (33) the stability condition can be written as $a_{0}>0, a_{1}>$ $0, a_{2}>0$.

We have $a_{0}>0$ trivially and since $\alpha, \mu^{(2)}$ and $\mu^{(1)}$ are positive so $a_{1}>0$.

Hence the condition of stability gives rise to $a_{2}>0$ and condition of marginal state is given by $a_{2}=0$ i.e.

$$
\left.\begin{array}{l}
\left(\rho^{(2)}-\rho^{(1)}\right) g-\sigma k^{2} \\
-2 k \alpha\left(\frac{\mu^{(1)}}{\rho^{(1)}} \operatorname{coth}\left(k h_{1}\right)+\frac{\mu^{(2)}}{\rho^{(2)}} \operatorname{coth}\left(k h_{2}\right)\right) \\
-\frac{k^{2} H_{0}^{2}\left(\mu_{m}^{(2)}-\mu_{m}^{(1)}\right)^{2}}{\left(\mu_{m}^{(1)} \operatorname{coth}\left(k h_{1}\right)+\mu_{m}^{(2)} \operatorname{coth}\left(k h_{2}\right)\right)}
\end{array}\right\}=0
$$

or

$$
\begin{aligned}
& k^{2}+\frac{k^{2} H_{0}^{2}\left(\mu_{m}^{(2)}-\mu_{m}^{(1)}\right)^{2}}{\left(\mu_{m}^{(1)} \operatorname{coth}\left(k h_{1}\right)+\mu_{m}^{(2)} \operatorname{coth}\left(k h_{2}\right)\right)} \\
& +\frac{2 k \alpha}{\sigma}\left(\frac{\mu^{(1)}}{\rho^{(1)}} \operatorname{coth}\left(k h_{1}\right)+\frac{\mu^{(2)}}{\rho^{(2)}} \operatorname{coth}\left(k h_{2}\right)\right)-k_{c}^{2}=0
\end{aligned}
$$

where $k_{c}$ is the well known classical wave-number, given by

$k_{c}^{2}=\frac{\left(\rho^{(2)}-\rho^{(1)}\right) g}{\sigma}$

If the fluids are inviscid i.e. $\mu^{(1)}=\mu^{(2)}=0$, heat and mass transfer has no effect on the stability of the system. Also if there is no heat and mass transfer across the interface i.e. $\alpha=0$, the inviscid potential flow (IPF) analysis and the viscous potential flow (VPF) solution predict the same critical wave number.

\section{Dimensionless form of the dispersion relation}

Introducing the dimensionless variables

$\hat{k}=k H, \quad \hat{\alpha}=\frac{\alpha H^{2}}{\mu^{(2)}}, \quad \hat{h}_{1}=\frac{h_{1}}{H}=\varphi$,

$\hat{h}_{2}=\frac{h_{2}}{H}=1-\hat{h}_{1}, \quad \hat{\rho}=\frac{\rho^{(1)}}{\rho^{(2)}}, \quad \hat{\mu}=\frac{\mu^{(1)}}{\mu^{(2)}}$,

$\hat{\mu}_{m}=\frac{\mu_{m}^{(1)}}{\mu_{m}^{(2)}}, \quad \hat{\omega}_{I}=\frac{\omega_{I} H}{Q} \quad \hat{H}=\frac{H_{0}}{Q} \sqrt{\frac{\mu_{m}^{(2)}}{\rho^{(2)}}}$

$\hat{\sigma}=\frac{\sigma}{\rho^{(2)} g H^{2}} \quad \vartheta=\frac{\mu^{(2)}}{\rho^{(2)} H Q}$

$\kappa=\frac{\hat{\mu}}{\hat{\rho}}, \quad \Lambda=\frac{\hat{\alpha} \vartheta^{2}}{\hat{\rho}}$

where $H=h_{1}+h_{2}$ and $Q=[((1-\hat{\rho}) g H) / \hat{\rho}]^{1 / 2}$

Here $\Lambda$ denotes alternate heat transfer coefficient and $\varphi$ represents the vapour thickness.

The dimensionless form of Eq. (33) can be expressed as $\left[\hat{\rho} \operatorname{coth}\left(\hat{k} \hat{h}_{1}\right)+\operatorname{coth}\left(\hat{k} \hat{h}_{2}\right)\right] \hat{\omega}_{I}^{2}+\vartheta\left[\hat{\alpha}\left(\operatorname{coth}\left(\hat{k} \hat{h}_{1}\right)+\operatorname{coth}\left(\hat{k} \hat{h}_{2}\right)\right)\right.$

$$
\begin{aligned}
& \left.+2 \hat{k}^{2}\left(\hat{\mu} \operatorname{coth}\left(\hat{k} \hat{h}_{1}\right)+\operatorname{coth}\left(\hat{k} \hat{h}_{2}\right)\right)\right] \hat{\omega}_{I} \\
& -\left[\hat{\rho} \hat{k}\left\{1+\frac{\hat{\sigma} \hat{k}^{2}}{(\hat{\rho}-1)}\right\}-2 \hat{k}^{2} \hat{\alpha} \vartheta^{2}\left\{\frac{\hat{\mu}}{\hat{\rho}} \operatorname{coth}\left(\hat{k} \hat{h}_{1}\right)+\operatorname{coth}\left(\hat{k} \hat{h}_{2}\right)\right\}\right. \\
& \left.-\frac{\hat{k}^{2} \hat{H}^{2}\left(1-\hat{\mu}_{m}\right)^{2}}{\left(\hat{\mu}_{m} \operatorname{coth}\left(\hat{k} \hat{h}_{1}\right)+\operatorname{coth}\left(\hat{k} \hat{h}_{2}\right)\right)}\right]=0
\end{aligned}
$$

The Eq. (37) contains the growth rate parameter $\vartheta=$ $\mu^{(2)} /\left(\rho^{(2)} h Q\right)$, which depends linearly on the kinematic viscosity $v^{(2)}=\mu^{(2)} / \rho^{(2)}$ of the upper fluid.

The Eq. (35) in the dimensionless form can be written as;

$$
\begin{gathered}
1+\frac{\hat{\sigma} \hat{k}^{2}}{(\hat{\rho}-1)}-2 \hat{k} \Lambda\left\{\kappa \operatorname{coth}\left(\hat{k} \hat{h}_{1}\right)+\operatorname{coth}\left(\hat{k} \hat{h}_{2}\right)\right\} \\
-\frac{\hat{k} \hat{H}^{2}\left(1-\hat{\mu}_{m}\right)^{2}}{\hat{\rho}\left(\hat{\mu}_{m} \operatorname{coth}\left(\hat{k} \hat{h}_{1}\right)+\operatorname{coth}\left(\hat{k} \hat{h}_{2}\right)\right)}=0
\end{gathered}
$$


From Eq. (38) we can also obtain the critical value of magnetic field $\hat{H}_{C}$. Thus the system is stable or unstable depending on whether the magnetic field is larger or smaller than $\hat{H}_{C}$.

\section{Results and discussions}

In this section, the numerical computation has been carried out using the expressions presented in the previous section for a film boiling condition. We consider vapour and water as working fluids identified with phase 1 and phase 2, respectively, such that $T_{1}>T_{0}>T_{2}$. The vapour can be treated as incompressible since the Mach number is expected to be small.

$\rho^{(1)}=0.001 \mathrm{~g} / \mathrm{cm}^{3}, \quad \rho^{(2)}=1.0 \mathrm{~g} / \mathrm{cm}^{3}$,

$\mu^{(1)}=0.00001$ poise, $\mu^{(2)}=0.01$ poise,

$\sigma=72.3$ dyne $/ \mathrm{cm}$

The effect of various physical parameters on the onset of instability is interpreted from the figures. Neutral curves divide the plane into a stable region (above the curve) and an unstable region (below the curve).

In Fig. 2, the neutral curves for the critical value of wave number with respect to the ratio of the kinematic viscosities of two fluids $\kappa$ have been drawn for the different values of alternate heat transfer coefficient $\Lambda$. The value of critical wave number has been obtained from Eq. (38) using Newton-Raphson method. It has been observed that as alternate heat transfer coefficient $\Lambda$ increases, the stable region also increases. As $\Lambda$ is directly proportional to the heat flux, therefore, the heat and mass transfer phenomenon stabilizes the classically unstable system. The heat and mass transfer plays stabilizing role in the absence of magnetic field as well

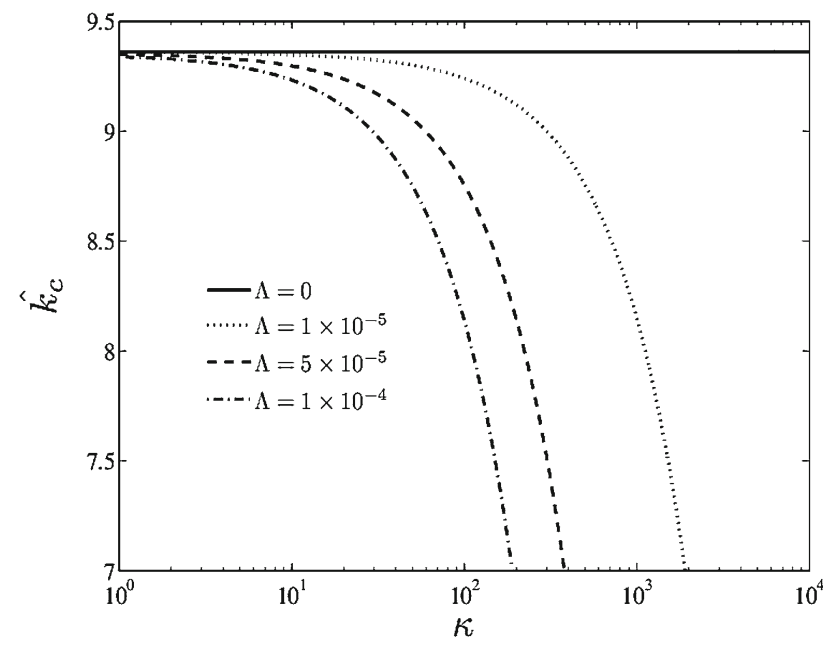

Fig. 2 Neutral curves for critical wave number system at $\hat{\rho}=0.001$, $\varphi=0.1, \hat{H}=0$ for the different values of alternative heat transfer coefficient $\Lambda$

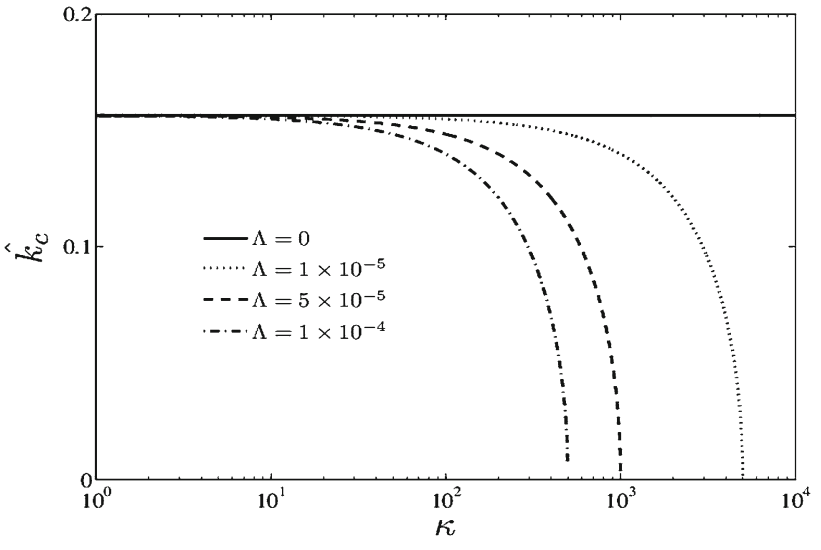

Fig. 3 Neutral curves for critical wave number system at $\hat{\rho}=0.001$, $\varphi=0.1, \hat{H}=1.0$ for the different values of alternative heat transfer coefficient $\Lambda$

(Awasthi et al. [21]).The effect of heat and mass transfer can be explained in terms of local evaporation and condensation at the interface. At a perturbed interface, crests are warmer because they are closer to the hotter boundary on the vapour side, thus local evaporation takes place, whereas troughs are cooler and thus condensation will take place. The liquid is protruding to a hotter region and the evaporation will diminish the growth of disturbance waves. Ozen and Narayanan [22] have given the comprehensive discussion of phase-change effects in interfacial stability. They have considered not only heat conduction but also convection. However, their system consisted of a liquid underlying its vapour between two parallel plates, where the plate in the liquid side is hotter. Figure 3 shows the effect of heat and mass transfer coefficient on the neutral curves of critical wave number in the presence of tangential magnetic field $\hat{H}=1.0$. It has been observed that the heat and mass transfer has still stabilizing effect on the stability of the system even in the presence of tangential magnetic field. From Figs. 2 and 3, it is very much clear that the stable region increases in the presence of magnetic field in comparison with the curves when there is no magnetic field. The magnetic field modifies the flow pattern and hence increases the rate of heat transfer across interface. The combination of magnetic field and heat transfer resists the growth of disturbance waves.

The effect of magnetic field strength $\hat{H}$ on the neutral curves of critical wave number has been considered in Fig. 4 when $\Lambda=10^{-4}$ and $\varphi=0.1$. It has been noticed that the increase in magnetic field strength, enlarges the stable region and therefore, magnetic field has stabilizing effect on the stability of the system. If magnetic field is present in the analysis, the term contributed from the applied magnetic field added in the right hand side of the Eq. (38) and so that critical value of wave number decreases. This shows that magnetic field has stabilizing influence. The concept of Lorentz force can elucidate the physical mechanism of this phenomenon. 


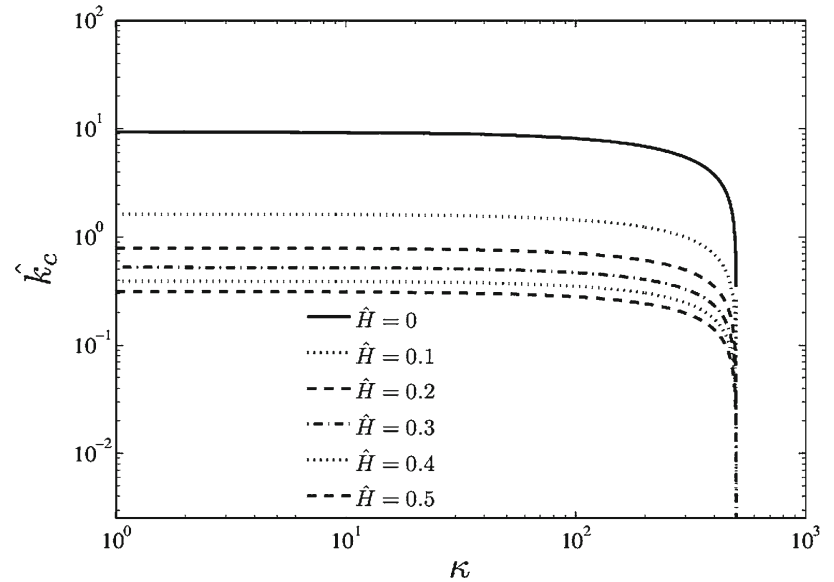

Fig. 4 Neutral curves for critical wave number system at $\hat{\rho}=0.001$, $\varphi=0.1, \Lambda=10^{-4}$ for the different values of magnetic field intensity $\hat{H}$

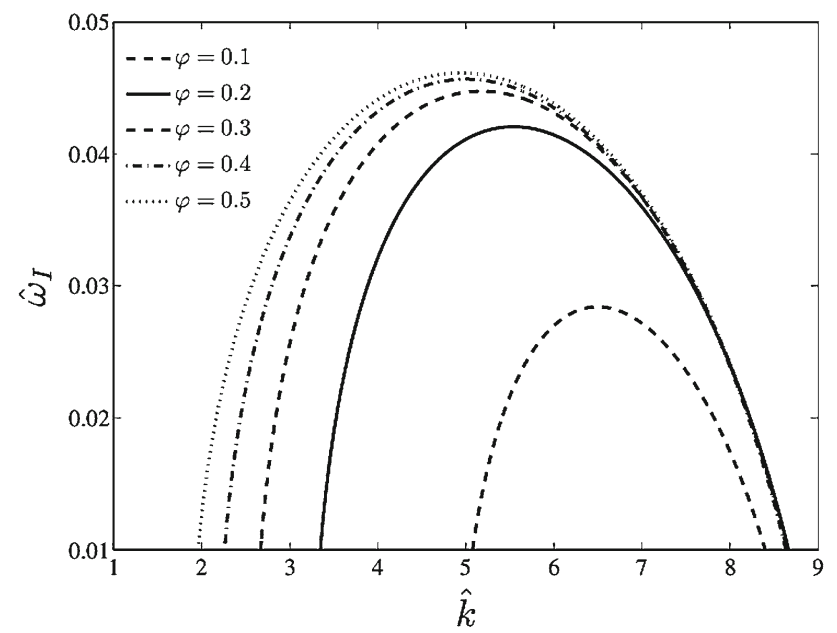

Fig. 5 Neutral curves for critical wave number system at $\hat{\rho}=0.001$, $\hat{\alpha}=1.0, \hat{H}=0.1$ for the different values of vapor fraction $\varphi$

Lorentz force due to the perturbed velocity and applied magnetic field has the effect of pushing the perturbed flow and hence, magnetic field has stabilizing effect.

Figure 5 shows the growth rate curves with respect to wave number for the different values of vapor thickness when the heat transfer coefficient $\hat{\alpha}=1.0$. The growth rate curves have been obtained from Eq. (38). The finding of Fig. 5 shows that as vapor thickness increases, growth rate increases and so vapor thickness plays a destabilizing role. As vapor thickness increases, at the crests more evaporation will take place. This additional evaporation will increase the amplitude of the disturbance waves and the system becomes destabilized.

In Fig. 6, variation of the growth rate curves $\hat{\omega}_{I}$ with wave number $\hat{k}$ has been shown for various values of magnetic field strength $\hat{H}$. It has been observed that as the magnetic field increases, growth rate decreases and this concludes that the tangential magnetic field $\hat{H}$ has stabilizing effect on the sta-

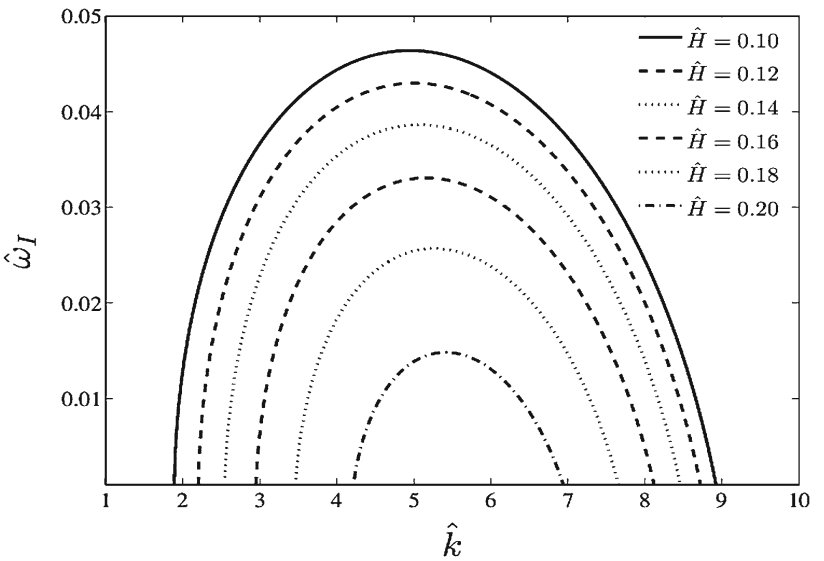

Fig. 6 Growth rate curves for different values of magnetic field when $\hat{\rho}=0.001, \hat{\alpha}=0.1, \varphi=0.5$

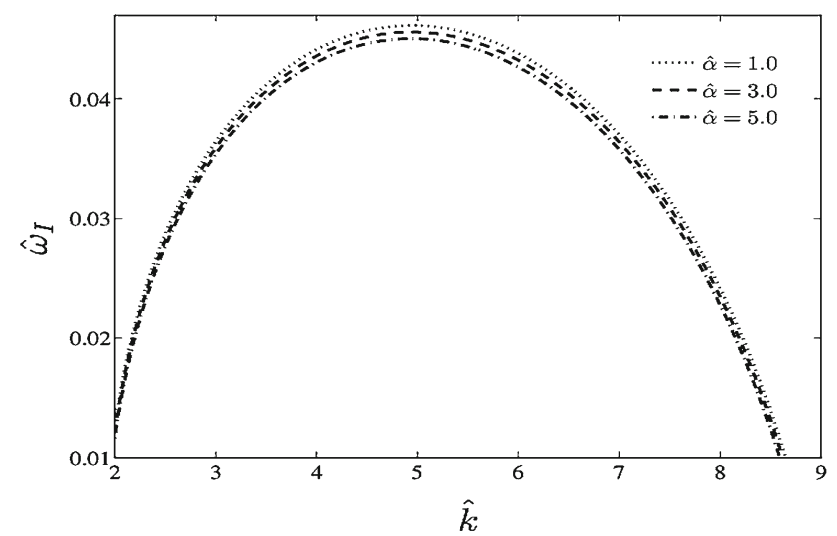

Fig. 7 Growth rate curves for different values of heat transfer coefficient when $\hat{\rho}=0.001, \hat{H}=0.1, \varphi=0.5$

bility of the system. The effect of heat transfer coefficient on the growth rate curves has been illustrated in Fig. 7. As heat transfer increases, the growth of disturbance waves decreases and hence the heat transfer phenomenon has stabilizing effect on the stability of the system.

In Fig. 8, the variation of maximum growth rate with the permeability ratio of two fluids $\hat{\mu}_{m}$ has been shown for heat transfer coefficient $\hat{\alpha}=1.0$ and vapour thickness $\varphi=0.5$. The Figure shows that as the ratio of the permeability's of the two fluids increases, the maximum growth rate first increases and then decreases. It shows that $\hat{\mu}_{m}$ has a dual role i.e. destabilizing as well as stabilizing effect on the stability of the system (Awasthi [23]). At the constant value of the magnetic field, the most unstable case was found when both the fluids have same magnetic permeability i.e. $\hat{\mu}_{m}=1$.

\section{Conclusion}

We study the viscous potential flow analysis of RayleighTaylor instability of two viscous and electrically conducting 


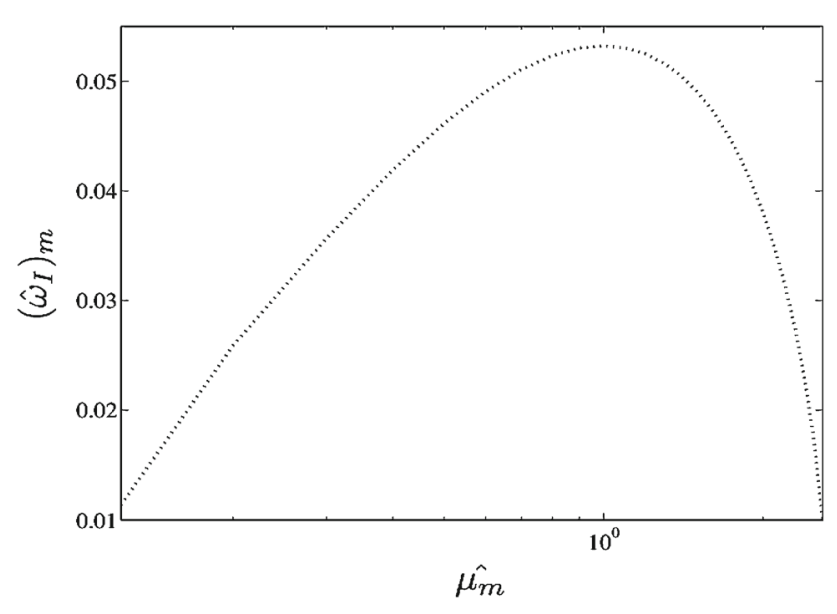

Fig. 8 Maximum growth rate curve versus magnetic permeability ratio when $\hat{\rho}=0.001, \hat{H}=0.1, \varphi=0.5, \hat{\alpha}=1.0$

fluids in the presence of tangential magnetic field when there is heat and mass transfer across the interface. The dispersion relation was a quadratic equation in the growth rate. The stability condition was given by Routh-Hurwitz criterion. A critical value of wave number as well as critical value of magnetic field was obtained. The system was shown to be unstable when the magnetic field is less than the critical value of magnetic field, otherwise it was stable. The heat and mass transfer, for inviscid fluids, has no effect on the stability of the system, while it has stabilizing effect on the stability for viscous fluids. It has been observed that the heat and mass transfer phenomenon has stabilizing effect on the stability of the system and this effect is enhanced in the presence of an axial magnetic field. The tangential magnetic field has stabilizing effect on the stability of the system while vapour fraction has destabilizing effect. The ratio of magnetic permeability of two fluids plays dual role in the linear stability analysis.

\section{References}

1. Chandrasekhar S (1981) Hydrodynamic and hydromagnetic stability. Dover, New York

2. Drazin PG, Reid WH (1981) Hydrodynamic stability. Cambridge University Press, Cambridge

3. Lewis DJ (1950) The instability of liquid surfaces when accelerated in a direction perpendicular to their planes.11. Proc R Soc Lond Ser A 201:81-96
4. Hsieh DY (1972) Effect of heat and mass transfer on RayleighTaylor instability. J Fluid Eng 94:156-162

5. Hsieh DY (1978) Interfacial stability with mass and heat transfer. Phys Fluids 21:745-748

6. Ho SP (1980) Linear Rayleigh-Taylor stability of viscous fluids with mass and heat transfer. J Fluid Mech 101:111-127

7. Khodaparast KA, Kawaji M, Antar BN (1994) The RayleighTaylor and Kelvin-Helmholtz stability of a viscous liquid-vapor interface with heat and mass transfer. Phys Fluids 7:359-364

8. Joseph DD, Liao T (1994) Potential flows of viscous and viscoelastic fluids. J Fluid Mech 256:1-23

9. Joseph DD, Belanger J, Beavers GS (1999) Breakup of a liquid drop suddenly exposed to a high-speed airstream. Int J Multiph Flow 25:1263-1303

10. Funada T, Joseph DD (2001) Viscous potential flow analysis of Kelvin-Helmholtz instability in a channel. J Fluid Mech 445:263283

11. Asthana R, Agrawal GS (2007) Viscous potential flow analysis of Kelvin-Helmholtz instability with mass transfer and vaporization. Phys A 382:389-404

12. Awasthi MK, Agrawal GS (2011) Viscous potential flow analysis of Rayleigh-Taylor instability with heat and mass transfer. Int J Appl Math Mech 7(12):73-84

13. Gill GK, Chhabra RK, Trehan SK (1995) Bubble formation in superposed magnetic fluids in the presence of heat and mass transfer. Z Naturforsch 50a:81-805

14. Moatimid GM, El-Dib YO (1996) Kelvin-Helmholtz instability of miscible ferrofluids. Int J Theor Phys 35:425-443

15. Elhefnawy ARF (1997) The nonlinear stability of mass and heat transfer in magnetic fluids. ZAMM 77:19-31

16. Moatimid GM (2002) On the stability of two rigidly rotating magnetic fluid columns in zero gravity in the presence of mass and heat transfer. J Colloid Interface Sci 250:108-120

17. Sirwah MA (2007) Nonlinear Kelvin-Helmholtz instability of magnetized surface waves on a subsonic gas-viscous potential liquid interface. Phys A 375:381-400

18. Sirwah MA (2008) Nonlinear temporal dynamics of two-mode interactions of magnetized flow. Int J Nonlinear Mech 43:416-436

19. Awasthi MK, Agrawal GS (2012) Viscous contributions to the pressure for potential flow analysis of magnetohydrodynamic KelvinHelmholtz instability. Int J Appl Mech 4:1-16

20. Awasthi MK, Asthana R, Agrawal GS (2012) Viscous corrections for the viscous potential flow analysis of magnetohydrodynamic Kelvin-Helmholtz instability with and mass transfer. Eur Phys J 48:174-183

21. Awasthi MK, Asthana R, Agrawal GS (2012) Viscous potential flow analysis of nonlinear Rayleigh-Taylor instability with heat and mass transfer. Microgravity Sci Technol 24:351-363

22. Ozen O, Narayanan R (2004) The physics of evaporative and convective instabilities in bilayer system: linear theory. Phys Fluids 16:4644

23. Awasthi MK (2013) Study on hydro-magnetic capillary instability with mass transfer through porous media. Int J Dyn Control 1:164171 